OPEN ACCESS

Edited by:

Sripathi Sureban,

University of Oklahoma Health

Sciences Center, United States

Reviewed by:

Ingo Schmidt-Wolf,

University Hospital Bonn, Germany

Hae Sun Suh,

Kyung Hee University, South Korea

*Correspondence: Hye-Lin Kim maristella76@tistory.com

Jeong-Hoon Lee

pindra@empal.com;

JHLeeMD@snu.ac.kr

${ }^{t}$ These authors have contributed equally to this work and share

first authorship

Specialty section:

This article was submitted to

Gastrointestinal Cancers,

a section of the journal

Frontiers in Oncology

Received: 22 June 2021 Accepted: 16 November 2021 Published: 03 December 2021

Citation:

Cho J-Y, Kwon S-H, Lee E-K, Lee J-H and Kim H-L (2021) CostEffectiveness of Adjuvant Immunotherapy With CytokineInduced Killer Cell for Hepatocellular Carcinoma Based on a Randomized Controlled Trial and Real-World Data.

Front. Oncol. 11:728740. doi: 10.3389/fonc.2021.728740

\section{Cost-Effectiveness of Adjuvant Immunotherapy With Cytokine- Induced Killer Cell for Hepatocellular Carcinoma Based on a Randomized Controlled Trial and Real-World Data}

\author{
Jeong-Yeon Cho ${ }^{1 \dagger}$, Sun-Hong Kwon ${ }^{1 \dagger}$, Eui-Kyung Lee ${ }^{1}$, Jeong-Hoon Lee ${ }^{2 *}$ \\ and Hye-Lin Kim ${ }^{3 *}$ \\ 1 School of Pharmacy, Sungkyunkwan University, Suwon, South Korea, ${ }^{2}$ Department of Internal Medicine and Liver \\ Research Institute, Seoul National University College of Medicine, Seoul, South Korea, ${ }^{3}$ College of Pharmacy, Sahmyook \\ University, Seoul, South Korea
}

Background: Studies using data from randomized controlled trials (RCTs) and real-world data (RWD) have suggested that adjuvant cytokine-induced killer (CIK) cell immunotherapy after curative treatment for hepatocellular carcinoma (HCC) prolongs recurrence-free survival (RFS) and overall survival (OS). However, the cost-effectiveness of CIK cell immunotherapy as an adjuvant therapy for HCC compared to no adjuvant therapy is uncertain.

Methods: We constructed a partitioned survival model to compare the expected costs, life-year (LY), and quality-adjusted life-year (QALY) of a hypothetical population of 10,000 patients between CIK cell immunotherapy and no adjuvant therapy groups. Patients with HCC aged 55 years who underwent a potentially curative treatment were simulated with the model over a 20-year time horizon, from a healthcare system perspective. To model the effectiveness, we used OS and RFS data from RCTs and RWD. We estimated the incremental cost-effectiveness ratios (ICERs) and performed extensive sensitivity analyses.

Results: Based on the RCT data, the CIK cell immunotherapy incrementally incurred a cost of $\$ 61,813,2.07 \mathrm{LYs}$, and 1.87 QALYs per patient compared to no adjuvant therapy, and the estimated ICER was $\$ 33,077 /$ QALY. Being less than the willingnessto-pay threshold of $\$ 50,000 / Q A L Y, C I K$ cell immunotherapy was cost-effective. Using the RWD, the ICER was estimated as $\$ 25,107 /$ QALY, which is lower than that obtained using RCT. The time horizon and cost of productivity loss were the most influential factors on the ICER. 


\begin{abstract}
Conclusion: We showed that receiving adjuvant CIK cell immunotherapy was more costeffective than no adjuvant therapy in patients with HCC who underwent a potentially curative treatment, attributed to prolonged survival, reduced recurrence of $\mathrm{HCC}$, and better prognosis of recurrence. Receiving CIK cell immunotherapy may be more costeffective in real-world clinical practice.
\end{abstract}

Keywords: cost-effectiveness, immunotherapy, adjuvant therapy, cytokine-induced killer cell, economic evaluation, hepatocellular carcinoma

\section{INTRODUCTION}

Hepatocellular carcinoma (HCC) is an aggressive and frequently occurring cancer, with approximately 670,000 new cases and 625,000 deaths worldwide in 2018 (1). Interestingly, most HCC cases occur in individuals with well-known risk factors, such as chronic hepatitis B virus (HBV) or hepatitis C virus (HCV) infection, nonalcoholic steatohepatitis, chronic alcoholism, and liver cirrhosis. Thus, a regular surveillance program for populations with such risk factors is recommended to detect HCC at an early stage, and more than half of new HCC cases are now diagnosed at very early or early stages in Japan and Taiwan owing to the implementation of nationwide surveillance programs (2).

Generally, in the very early or early stages of HCC, potentially curative treatments, such as surgical resection or radiofrequency ablation (RFA), can be applied; however, even after successful resection, $50-70 \%$ of patients experience recurrence within 5 years $(3,4)$. The long-term prognosis of patients with recurrent HCC remains poor because of deterioration of liver function with repeated recurrences even after curative treatment following early detection of HCC and during a successful regular surveillance program (5). Therefore, in the treatment of HCC, recurrence after curative treatment is an important indicator that is negatively related to long-term survival (6). To improve recurrence-free survival (RFS) in patients with HCC, several studies on adjuvant therapy have been conducted; however, none of these studies provide sufficient evidence of improvement except for antiviral treatment for HBV- or HCV-related HCC (3).

Since the first report on the antitumor activity of cytokineinduced killer (CIK) cells, 106 clinical trials for various types of cancers have been registered in the international registry of CIK cells in the past decade $(7,8)$. Fortunately, recent phase III trials and real-world data (RWD) have shown that adjuvant CIK cell immunotherapy administered after curative treatment for HCC prolongs RFS, cancer-specific survival, and overall survival (OS) with minimum adverse effects. According to previous studies, patients who underwent repeated transfer of individualized autologous CIK cell agents had significantly longer RFS than the control group with a hazard ratio of 0.42 (95\% confidence interval [CI], 0.22-0.80) to 0.63 (95\% CI, 0.43-0.94) (9-11). These results have generated interest in the economic assessment of the benefits obtained and the input cost for adjuvant CIK immunotherapy. Nevertheless, there is limited data on the costeffectiveness of immunotherapy in patients with HCC who have undergone potentially curative treatment.
Therefore, it is important for healthcare policymakers, providers, and patients to determine whether adjuvant CIK immunotherapy reflects an appreciable value in the current healthcare environment. We investigated the cost-effectiveness of CIK cell immunotherapy as an adjuvant therapy for patients with HCC using data from a recently reported phase III trial and RWD.

\section{MATERIALS AND METHODS}

\section{Overview of Partitioned Survival Model}

A cost-utility analysis was performed to evaluate the costeffectiveness of receiving CIK cell immunotherapy compared with not receiving any adjuvant treatment (no adjuvant therapy) in patients with HCC who underwent a potentially curative treatment (surgical resection, RFA, or percutaneous ethanol injection $[\mathrm{PEI}]$ ). Aligning with the phase III clinical study (the randomized controlled trial, hereafter referred to as RCT), its extended follow-up study $(9,10)$, and the phase IV clinical study (hereafter referred to as RWD) of CIK cell immunotherapy (11), we constructed a partitioned survival model, which has been widely used for the economic evaluation of oncology drugs, using Microsoft Excel (Microsoft, Redmond, Washington, USA) (12). The time horizon of the model was twenty years, and the cycle length was three months.

Our model included four conceptual health states: recurrence-free, curable recurrence, incurable recurrence, and death (Figure 1A). Because our population included patients with HCC who underwent a potentially curative treatment, all patients started from the recurrence-free state. RFS was defined as the time from randomization in RCT (in RWD, from curative treatment) to the first recurrence or death. Patients without recurrence remained in the recurrence-free state as the cycle went on. Otherwise, the patient experienced recurrence or death. Unlike a typical partitioned survival model, we divided the recurrence condition into two states according to the curability of treatment to consider the different treatments patients undergo. We assumed that patients received either curative or noncurative treatments once they demonstrated a recurrence. If patients remained in the curable recurrence state, they were treated with curative treatments, such as surgical resection, liver transplantation, RFA, or PEI. By contrast, patients staying in an incurable recurrence state would receive noncurative treatments including transarterial chemoembolization (TACE), external- 
A Conceptual model

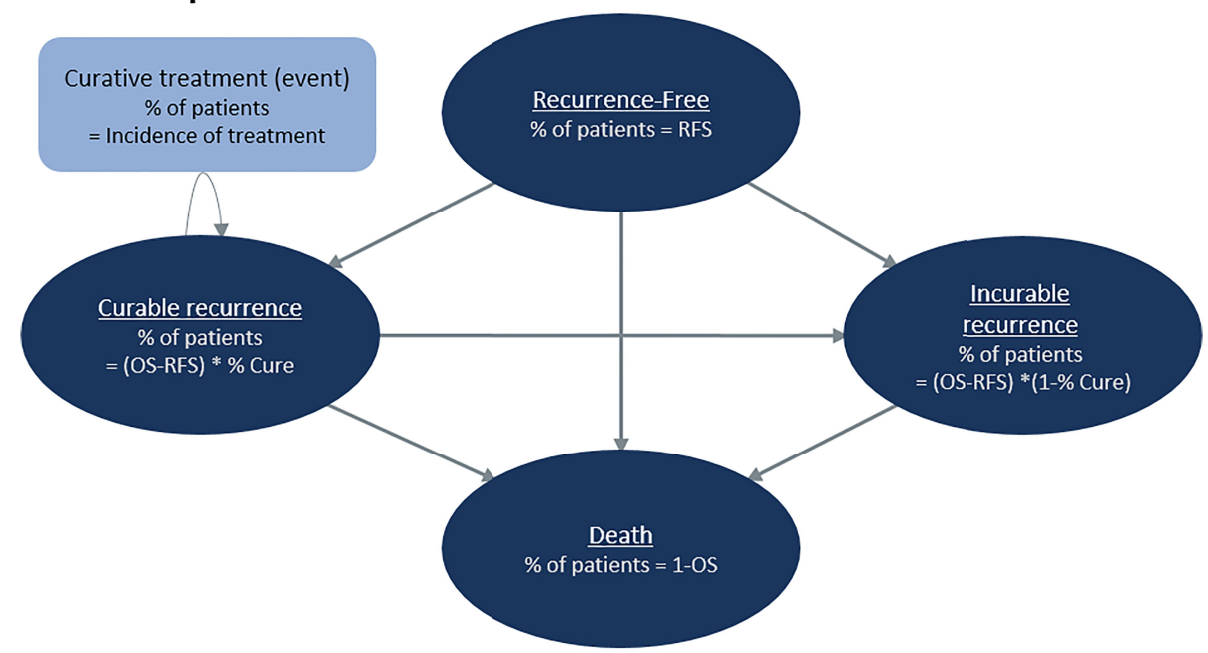

B Partitioned survival model
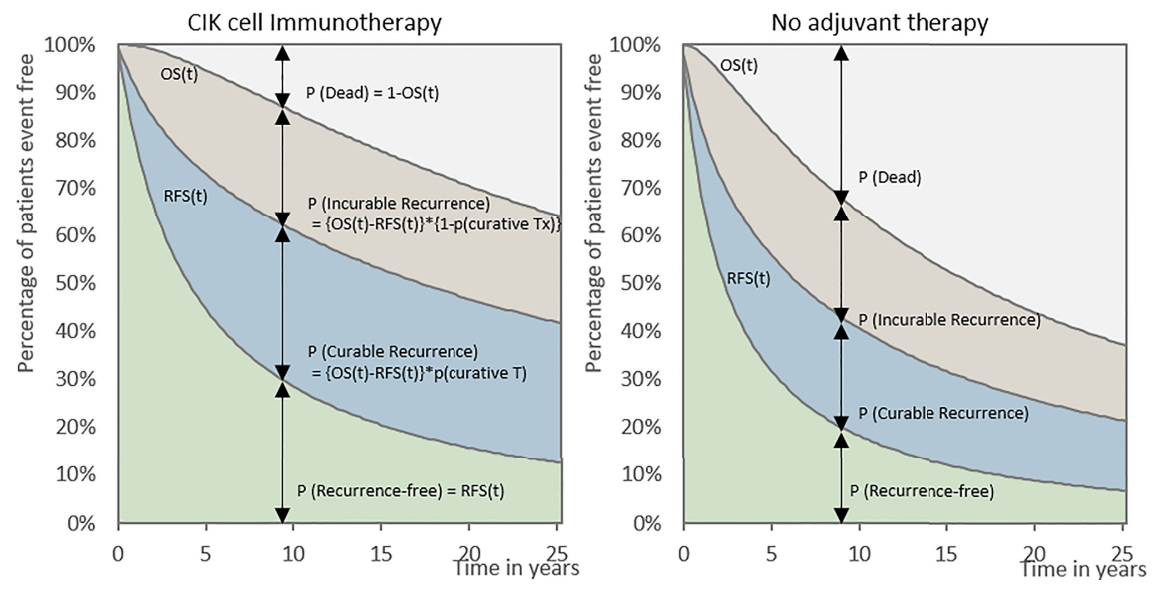

FIGURE 1 | Cost-effectiveness model. (A) Conceptual model and (B) partitioned survival model. OS, overall survival; RFS, recurrence-free survival.

beam radiation therapy, and systemic therapies including cytotoxic chemotherapy. We performed a post-hoc analysis of the records of individual patients on subsequent treatment and follow-up time after recurrences observed in phase III clinical trials to calculate the proportion of patients with curable recurrence and the incidence rates of each curative treatment applied in the curable recurrence state. For the treatment of HCC, liver transplantation, surgical resection, or RFA is considered as potentially curative treatment. In the base-case analysis, according to the international guideline, we assumed that once the patient received other non-curative therapy, such as TACE or systemic therapies including cytotoxic chemotherapy, the recurrence was classified as incurable (13). However, some of these patients might have received TACE for curative intent as curative treatment was not technically feasible; therefore, we adjusted the proportion of curative TACE to the calculation of curable recurrence in the sensitivity analysis. Our model simulated a cohort of 55-year-old patients, considering the mean age of patients in the RCT (9). To validate our model, clinical experts verified the key model assumptions.

The model estimated the costs, gained life-years (LYs), and quality-adjusted life-years (QALYs) for a 20-year time horizon. An annual discount rate of 5\% was applied to the outcomes and costs, according to the pharmacoeconomic evaluation guideline from the Health Insurance Review \& Assessment Service (HIRA) of the South Korean government (14). This economic evaluation was conducted and reported based on the Consolidated Health Economic Evaluation Reporting Standards (CHEERS) guidelines (15).

\section{RFS and OS}

Two survival curves of RFS and OS derived from the RCTs and RWD were applied in the model to estimate the distribution of patients in modeled health states following CIK cell immunotherapy or no adjuvant therapy (9-11). Using the effectiveness data from the RWD, we tried to reflect current 
clinical practice for the early stage of HCC and to reduce the gap between the RCTs and the real-world setting Both RCT and RWD routine surveillance with CIK cell immunotherapy in an adjuvant setting for HCC were compared. At the median followup of 68.5 months for RCT and 28.0 months for RWD, investigators found statistically and clinically significant improvements in RFS (9-11). The patient characteristics and main results of both RCT and RWD were summarized in Supplementary Table 1.

RFS at a specific time was defined as the proportion of patients staying in the recurrence-free state (Figure 1B). The fraction of patients with recurrence was the difference between the OS and RFS (OS-RFS). The proportions of curable and incurable recurrence states were obtained from the RCTs to differentiate between patients receiving curative and noncurative treatment (Table 1). The incidence of curative treatment was estimated from the individual patient data reported in the RCTs. With regard to the distribution of patients who died, [1-OS] was adapted, as shown in Figure 1B.

To extrapolate patient survival beyond the duration of the clinical trial, parametric survival curves were fitted to our model: exponential, Weibull, generalized gamma, log-normal, and loglogistic. They were modeled jointly for each treatment group, and the best fit was determined using the Akaike information criterion, Bayesian information criterion, plausibility of the estimated long-term survival, and expert opinion (Supplementary Table 2 and Supplementary Figures 1, 2) $(12,19)$. The parameter estimates for survival curves are presented in Supplementary Table 3.

\section{Cost and Utility}

We considered direct medical costs, which were represented in the model either as episodic costs for curative treatments and death (end-of-life) or state-based costs, in terms of the healthcare system perspective. The input costs, presented in USD in 2020 (1 USD $=1,166.51 \mathrm{KRW})$, are shown in Table 1 . The cost data before 2020 were corrected for inflation using the national inflation calculator.

Treatment-specific costs in the recurrence-free state were estimated based on healthcare utilization from RCT and the unit cost from the reimbursement price list from HIRA. In accordance with the label of CIK cell immunotherapy,

TABLE 1 | Model input parameters.

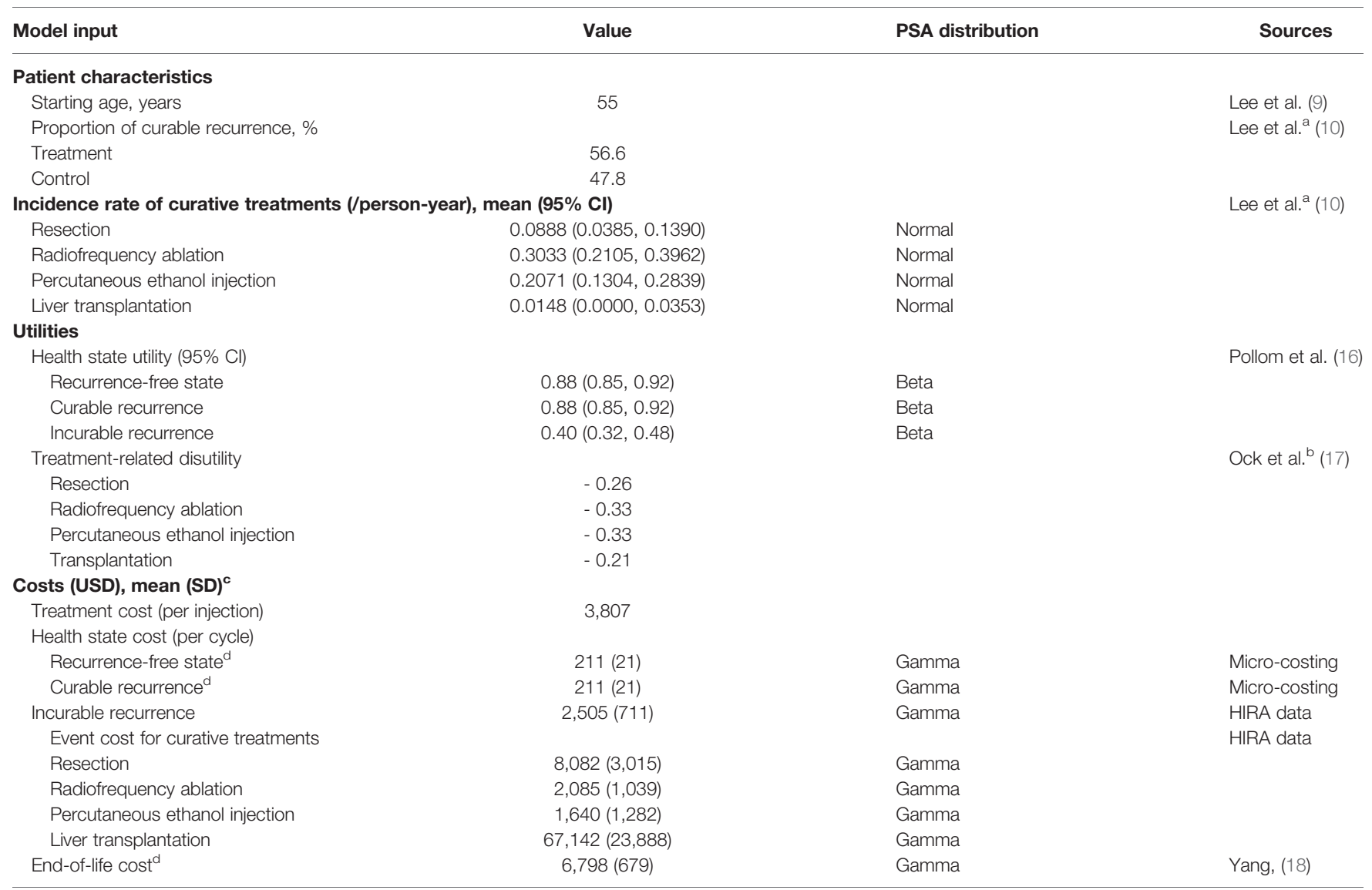

Cl, confidence interval; HIRA, Health Insurance Review \& Assessment Service of Korea; PSA, probabilistic sensitivity analysis; SD, standard deviation.

${ }^{a}$ Derived from post-hoc analysis of recurrence data from phase III trial (Lee et al., 2015 \& Lee et al., 2018).

${ }^{b}$ Converted to disutility from Ock et al., 2017.

${ }^{c} 1 \mathrm{USD}=1,166.51 \mathrm{KRW}$.

${ }^{d}$ Standard deviation is assumed to be $10 \%$ of the mean value. 
treatment cost was considered for a total of 16 times at the price proposed by the manufacturer.

The medical costs for recurrence were obtained from the HIRA National Patient Sample (HIRA-NPS-2016-0106) data. The cost analysis of HIRA-NPS data was approved by the institutional review board of Sungkyunkwan University. The HIRA-NPS data are representative of the South Korean population, which includes approximately 3\% of the total population (20, 21). From the HIRA-NPS data, we extracted the medical costs per episode of each curative treatments (i.e., resection, RFA, PEI, and transplantation) and noncurative treatments (i.e., TACE, external-beam radiation therapy, and systemic therapy with sorafenib or cytotoxic chemotherapy) from patients with the main diagnosis code of HCC (C22.0 of ICD-10, International Classifications of Disease 10th version) in 2016. The detailed procedure codes which were used to estimate the medical costs for each treatment were presented in Supplementary Table 4 . For the curable recurrence state, the cost for each curative treatment was individually applied according to the incidence of curative treatment in the model. For the incurable recurrence state, the state-based cost was calculated by multiplying the cost of noncurative treatment by the frequency obtained from the RCT. The end-of-life costs were adapted from a previous study that observed end-of-life costs for patients with cancer (18).

We performed a systematic literature review using PubMed and the Cochrane library to obtain utility values for each health state in our model $(16,17)$. Utility is a number between 0 (death) and 1 (perfect health), which is used to calculate QALY by taking length and quality of life into consideration (22). A detailed list of the utilities and disutilities included in the model are shown in Table 1.

\section{Analysis}

The main output of this study was the incremental costeffectiveness ratio (ICER), which was calculated by dividing the incremental costs by the incremental LYs and QALYs between the CIK cell immunotherapy group and no adjuvant therapy group. The cost-effectiveness was interpreted using ICER at the willingness-to-pay (WTP) threshold of \$50,000/QALY.

Furthermore, one-way deterministic and probabilistic sensitivity analyses were performed to explore the robustness of the results by varying the parameter values and assumptions. For the one-way sensitivity analysis, clinical variables (parametric survival distribution to OS and RFS, and the proportion of patients receiving curative treatment), utility weights, medical costs, analytic perspective, time horizon, and discount rate were changed. Medical costs in other countries were also applied to the sensitivity analysis and are presented in Supplementary Table 5 (23-25).

For the probabilistic sensitivity analysis, a comprehensive estimate of the uncertainty around the results was calculated using simultaneous random sampling of input parameters. The range and distribution of the parameters are listed in Table $\mathbf{1}$ and Supplementary Table 2 . The probabilistic sensitivity analysis consisted of 1,000 iterations with random values generated according to the range or distribution of each parameter included in the model. In addition, a cost-effectiveness acceptability curve (CEAC) was constructed to show the likelihood of the intervention being cost-effective according to the various WTP thresholds.

\section{RESULTS}

Based on the RCT data, the CIK cell immunotherapy resulted in 11.68 LYs and 8.80 QALYs per patient costing \$115,002, whereas no adjuvant therapy resulted in 9.60 LYs and 6.94 QALYs per patient costing $\$ 53,190$ (Table 2). Throughout the time horizon, the incremental LYs was 2.07 years, and the patients treated with CIK cell immunotherapy remained in the recurrence-free state longer than the patients without treatment ( 5.43 vs. 4.07 years, Figure 2). For treatment costs, a substantial difference between the two interventions was observed in the recurrence-free state. The incremental QALYs was 1.87 costing $\$ 61,813$, and the ICER was $\$ 33,077 /$ QALY.

Based on the RWD, the CIK cell immunotherapy resulted in 12.53 LYs and 9.76 QALYs per patient with a treatment cost of $\$ 110,670$, whereas no adjuvant therapy resulted in 10.68 LYs and 7.66 QALYs per patient costing $\$ 57,959$. The incremental lifeyears gained was 1.85, and the incremental QALY was 2.10, resulting in an ICER of \$25,107/QALY. The ICERs estimated based on RCT data and RWD were both less than $\$ 50,000$, which showed the cost-effectiveness of CIK cell immunotherapy.

The results of the deterministic sensitivity analysis are presented in Supplementary Figure $\mathbf{3}$ and Table 3, showing the 10 most sensitive input parameters, given RCT data and RWD. The parameter that most influenced the ICER was the

TABLE 2 | Result of the cost-effectiveness analysis.

\begin{tabular}{|c|c|c|c|c|}
\hline & CIK cell Immunotherapy & No adjuvant therapy & Incremental & ICER (\$/LY or \$/QALY) \\
\hline \multicolumn{5}{|c|}{ Based on RCT data } \\
\hline Cost & $\$ 115,002$ & $\$ 53,190$ & $\$ 61,813$ & \\
\hline LY & 11.68 & 9.60 & 2.07 & $\$ 29,791$ \\
\hline QALY & 8.80 & 6.94 & 1.87 & $\$ 33,077$ \\
\hline \multicolumn{5}{|c|}{ Based on RWD } \\
\hline Cost & $\$ 110,670$ & $\$ 57,959$ & $\$ 52,711$ & \\
\hline LY & 12.53 & 10.68 & 1.85 & $\$ 28,437$ \\
\hline QALY & 9.76 & 7.66 & 2.10 & $\$ 25,107$ \\
\hline
\end{tabular}

CIK, cytokine-induced killer cell; LY, life-year; QALY, quality-adjusted life-year; ICER, Incremental cost-effectiveness ratio; RCT, randomized controlled trial; RWD, real-world data. 


\section{A Cost}

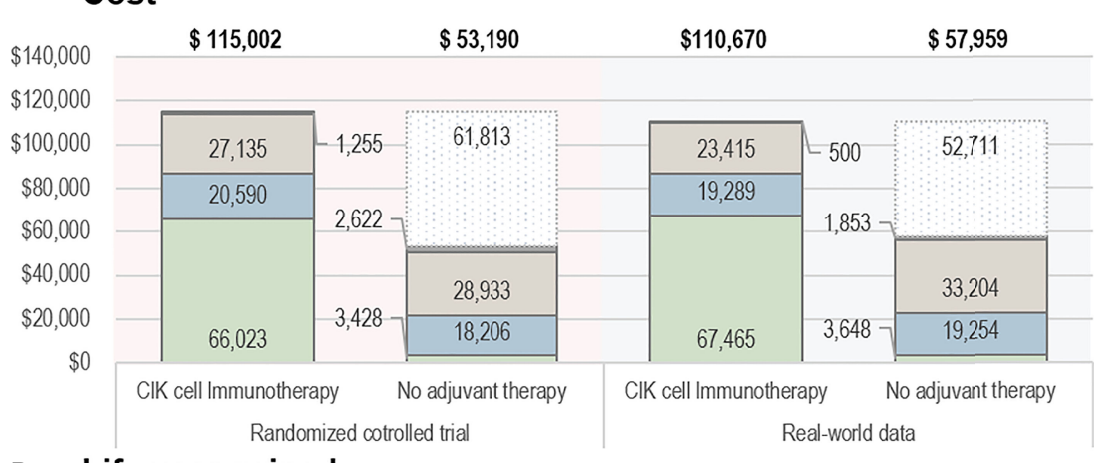

B Life year gained

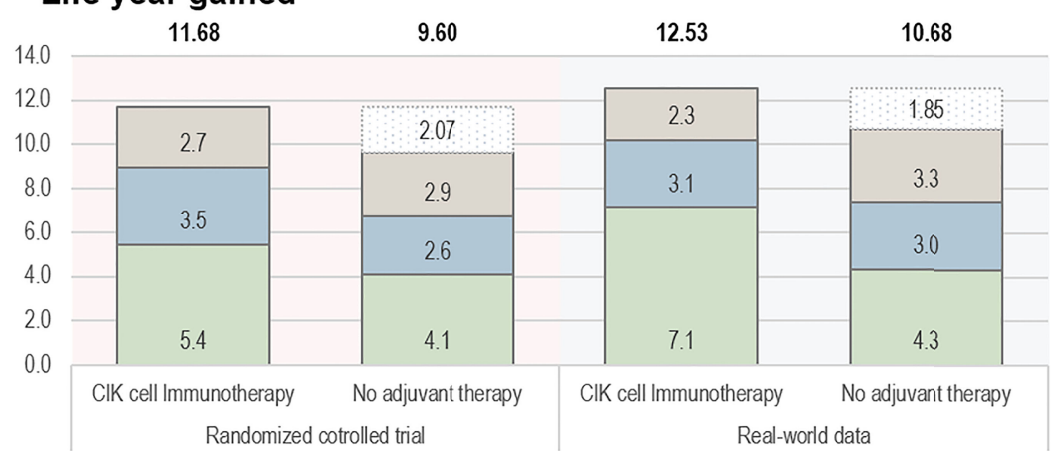

C Quality-adjusted life year gained

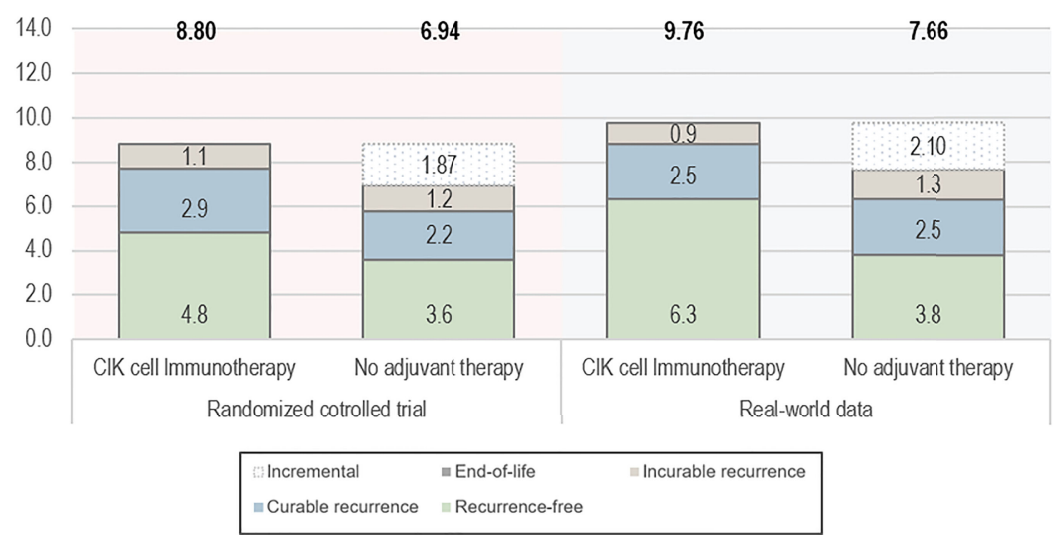

FIGURE 2 | Base-case results. (A) Cost, (B) life-year gained, and (C) quality-adjusted life-year gained. The bold text indicates the total value estimated from the analyses. CIK, cytokine-induced killer.

time horizon. Regarding other parameters, including productivity loss in the analysis (societal perspective) resulted in lower ICER $(\$ 25,562)$, whereas applying medical costs from Italy and the USA showed the robustness of the study results with ICER ranging from $\$ 34,141-\$ 38,425$ (Supplementary Table 3). All ICERs based on the RWD were lower than those based on the RCTs. The CEAC calculated from our model showed that the likelihood of CIK cell immunotherapy being cost-effective was $95 \%$ and $88 \%$ based on RCT and RWD, respectively, with a WTP threshold of $\$ 50,000$ (Figure 3). For WTP values below $\$ 42,350$, the cost-effectiveness acceptability based on RWD was higher than that based on RCT, but the trend reversed for WTP values beyond $\$ 42,350$.

\section{DISCUSSION}

This study demonstrated that adjuvant CIK cell immunotherapy in patients who received curative treatment for HCC is costeffective as compared with no adjuvant therapy. By applying data from RCTs and an extended follow-up study, we showed that the higher LYs and QALYs gained from receiving CIK cell 
TABLE 3 | Deterministic sensitivity analyses..

\begin{tabular}{|c|c|c|}
\hline & \\
\hline & RCT & RWD \\
\hline \multicolumn{3}{|l|}{ Clinical outcomes } \\
\hline RFS [Worst case; Log-normal (RCT), Generalized gamma (RWD)] & 33,077 & 31,695 \\
\hline OS [Best case; Weibull (RCT), Generalized gamma (RWD)) & 31,009 & 22,587 \\
\hline OS [Worst case; Generalized gamma (RCT), Log-normal (RWD)] & 38,831 & 27,545 \\
\hline \multicolumn{3}{|l|}{ Health-related quality of life } \\
\hline \multicolumn{3}{|l|}{ Health state utilities } \\
\hline Cancer free and incurable recurrence state (Upper bound) & 31,876 & 24,978 \\
\hline Cancer free and incurable recurrence state (Lower bound) & 33,971 & 25,236 \\
\hline \multicolumn{3}{|l|}{ Costs } \\
\hline \multicolumn{3}{|l|}{ Medical costs from other healthcare systems } \\
\hline Upper bound (+20\%) & 32,930 & 24,695 \\
\hline Lower bound $(-20 \%)$ & 33,223 & 25,187 \\
\hline \multicolumn{3}{|l|}{ Analytic perspective (Societal perspective) } \\
\hline Including productivity loss cost & 25,562 & 19,858 \\
\hline \multicolumn{3}{|l|}{ Model parameters } \\
\hline Time horizon (15 years) & 41,628 & 32,730 \\
\hline Time horizon (25 years) & 28,799 & 21,263 \\
\hline Annual discount rate (3\%) & 27,617 & 20,336 \\
\hline Annual discount rate (7.5\%) & 40,926 & 31,973 \\
\hline
\end{tabular}

ICER, incremental cost-effectiveness ratio; RCT, randomized controlled trial; RWD, real-world data; RFS, recurrence-free survival; OS, overall survival; TACE, transarterial chemoembolization. ${ }^{*}$ To see the uncertainty from the selected survival curve, we carried out a sensitivity analysis that calculated the lowest (best case) and the highest (worst case) ICER by applying each parametric survival distribution to OS and RFS.

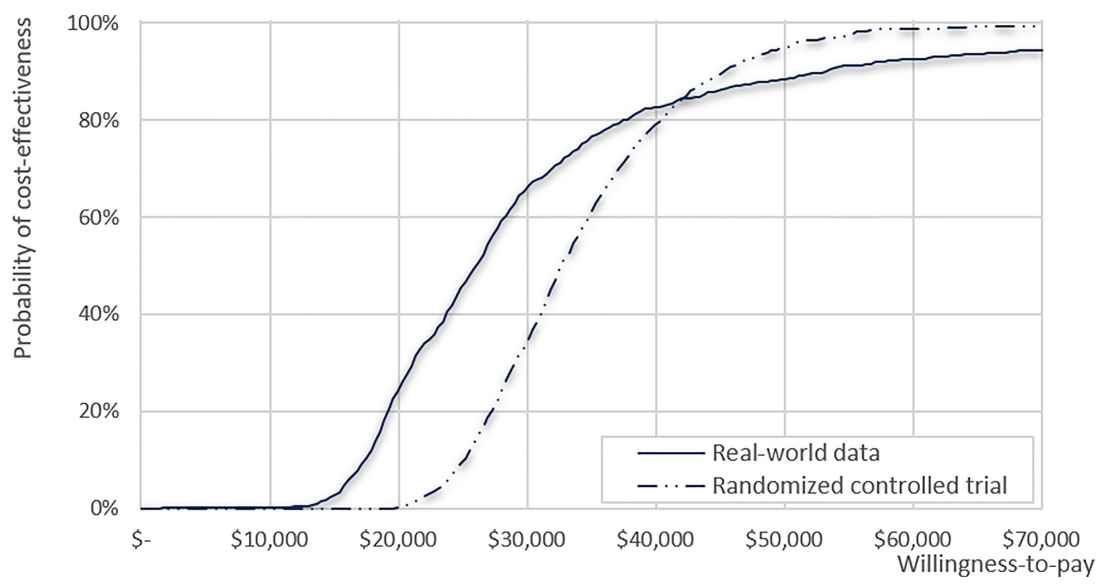

FIGURE 3 | Cost-effectiveness acceptability curve for clinical data from the real-world data and randomized clinical trial.

immunotherapy compared with no adjuvant therapy resulted from prolonged survival, reduced recurrence of HCC, and better prognosis of recurrence $(9,10)$. Furthermore, decreased medical expenses for the treatment of incurable recurrent HCC partially offset the considerable treatment cost of CIK cell immunotherapy in patient with curable recurrent HCC.
Consequently, adjuvant CIK cell immunotherapy could be a cost-effective option based on a WTP threshold of US\$ 50,000/QALY.

The simulation results using RWD, where CIK cell immunotherapy prolonged the RFS of patients with HCC, showed further improvement in cost-effectiveness, which was 
attributed to more favorable survival for CIK cell immunotherapy than that from the RCT data. The costeffectiveness model using RWD shows that more patients stayed in recurrence-free health state due to better RFS than that from RCT. Therefore, scenario using RWD incurred less medical expenditure and have more health-related quality of life due to prolonged recurrence-free survival, which means the costeffectiveness results using RWD were better than that from RCT data.

In the RCT, adjuvant CIK cell immunotherapy was more effective at reducing the rate of early recurrence within the first 24 months (which is mainly associated with residual tumor cells) than late recurrence beyond 24 months (which is mainly related to de novo carcinogenesis from diseased liver). Based on this result, the authors of the RWD study suggested that a shorter follow-up duration of RWD (28.0 months) than that of RCT (68.5 months) may be associated with lower HR of tumor recurrence ( 0.42 vs 0.67$)$ (9-11). However, it should be noticed that the baseline characteristics of patients were worse with higher tumor stage and larger tumor size in RWD than in RCT. These baseline characteristics might be unfavorable to RWD as CIK cell treatment is expected to be more effective in patients with less tumor burden (9). Thus, the lower HR of tumor recurrence or death in RWD is not simply explainable with currently available data and further real-world studies are warranted.

Our findings provide evidence for generalizing the effectiveness of RCTs conducted in controlled populations in the real-world population. The participants in the RCT, who were included following clear inclusion and exclusion criteria and treated following a well-defined schedule, may not represent patients in real-world practice (26). Thus, the validation of the effectiveness of CIK cell adjuvant therapy in RWD is essential, and the benefit of RFS is reproducible. Furthermore, our findings are meaningful in that a more beneficial economic value of CIK cell immunotherapy could be obtained in real-world clinical practice. In addition, the cost-effectiveness results from both RCT and RWD can support decision-making on introducing CIK cell immunotherapy in an adjuvant setting.

The analysis conducted under the societal perspective considering productivity loss was remarkable compared to that conducted from the healthcare system perspective. HCC occurs more frequently in men and is most frequently diagnosed among people aged 55-64 years who are economically active, and also occurs at a younger age, especially in Asia and sub-Saharan Africa, where $\mathrm{HBV}$ is endemic, than in other regions where $\mathrm{HBV}$ is not a predominant etiology of HCC (27-30). As the socioeconomic burden of disease because of premature death is substantial, preventing recurrence in such patients would reduce productivity loss in society. In South Korea, an HBV-endemic country, HCC was the second-highest cause of cancer-related death (21.5 per 100.000 population) in 2016 following lung cancer but resulted in the highest economic burden (USD 3.144 million) in 2010 among all cancers (31). It is a disappointing outcome considering that the nationwide regular surveillance program is now working well and approximately
$40 \%$ of new HCC cases are diagnosed at a very early or early stage, where potentially curative treatment can be applied (32). Accordingly, the urgent need for cost-effective adjuvant therapy should be highlighted again.

Our findings on the cost-effectiveness of CIK cell immunotherapy, which prevents the recurrence of HCC, align with the results of previous cost-effectiveness studies on adjuvant therapies. Although there is no guideline-suggested adjuvant therapy for patients with HCC, several RCTs were conducted for adjuvant therapy with an effective drug used in the management of advanced HCC, such as sorafenib $(33,34)$. Because our study was the first to demonstrate the costeffectiveness of treatment to prevent recurrence in HCC, there was no previous study to report the cost-effectiveness of adjuvant therapy in patients with HCC who underwent a potentially curative treatment. However, there have been several studies on other types of cancers, in which cost-effectiveness was verified by preventing recurrence or relapse (35-37). Our results emphasize the importance of preventing recurrence after successful primary therapy, which is in line with previously reported findings.

Our research has significant strengths, although decisionanalytic models have limitations regarding the input parameters applied in the model. First, our original model was designed to reflect the prognosis of HCC recurrence, which is not generally considered in the conventional three-state model. To reflect the heterogeneous prognosis of recurrence, we conducted a post-hoc analysis of subsequent treatments (curative and noncurative treatments) from the phase III clinical trial, followed by the proportion of curable recurrence that we applied to our original model. Therefore, we have improved the model plausibility by reflecting the different prognoses of recurrence and healthrelated quality of life of patients. In addition, we demonstrated the robustness of cost-effectiveness by performing probabilistic and deterministic sensitivity analyses. Most of the input parameters were derived from the patient-level data of the phase III trial, and the remaining input parameters were verified by clinical experts. Moreover, we applied a wide range of costs and effectiveness to our model to assess inherent uncertainties.

Although our study considered various ranges of uncertainty, there were some limitations. First, the applied costs were obtained from the Korean healthcare system. Therefore, the costs were potentially lower than those in other developed countries, such as the USA. However, cost-effectiveness evaluations focus on the 'incremental' costs and effectiveness of CIK cell immunotherapy, and because the unit medical costs affected both the intervention and comparator in our model, the impact of lower costs in South Korea on the results would be limited. Even if the local cost data were replaced with those in other developed countries, including the USA, France, and Italy, the estimated ICERs from those scenarios $(\$ 34,000-\$ 38,000 /$ QALY) were similar to our base-case analysis results and were similar to or lower than each country's gross domestic product (GDP) per capita. Because the cost-effectiveness threshold for anticancer drugs is generally accepted as approximately twice the 
GDP per capita, CIK cell immunotherapy would be a costeffective alternative in all of these countries as well. In addition, the discount rate applied to our model was $5 \%$, which was slightly higher than that in other developed countries; thus, the cost-effectiveness of CIK cell immunotherapy was analyzed conservatively because the longterm effectiveness and offset treatment costs of incurable recurrent HCC were underestimated in the base-case setting.

Second, survival data from RWD study has a relatively shorter observation period than RCTs, and there were some differences in the baseline characteristics among RWD and RCTs. This may increase the uncertainty of modeled survival output using RWD. Therefore, we simulated various parametric survival functions in sensitivity analyses to alleviate the uncertainty caused by short observation periods and different patient characteristics from RCTs, and presented the minimum and maximum values of ICER according to various survival functions in the tornado diagram (Supplementary Figure 3). As a result, our model showed robust results even when using RWD data. Finally, the proportion of curable recurrent HCC was calculated based on operational definitions using the subsequent treatment data of patients obtained from RCTs. For example, each recurrence treated with TACE was classified as incurable recurrence. However, there could be a curative TACE for patients who are not eligible for other curative treatments, which could lead to an underestimation of the proportion of curable recurrence. Even if we adopted the most generous criteria for classifying the curability of recurrence, the ICER increased by only $7 \%$ from the base-case analysis, and CIK cell immunotherapy was still cost-effective.

In conclusion, we showed that receiving CIK immunotherapy was more cost-effective than no adjuvant therapy in patients with HCC who underwent a potentially curative treatment, attributed to the prolonged survival, reduced recurrence of HCC, and better prognosis of recurrence, and it could be even more cost-effective in the real-world clinical practice.

\section{REFERENCES}

1. Bray F, Ferlay J, Soerjomataram I, Siegel RL, Torre LA, Jemal A. Global Cancer Statistics 2018: GLOBOCAN Estimates of Incidence and Mortality Worldwide for 36 Cancers in 185 Countries. CA Cancer J Clin (2018) 68 (6):394-424. doi: 10.3322/caac.21492

2. Park JW, Chen M, Colombo M, Roberts LR, Schwartz M, Chen PJ, et al. Global Patterns of Hepatocellular Carcinoma Management From Diagnosis to Death: The BRIDGE Study. Liver Int (2015) 35(9):2155-66. doi: 10.1111/liv.12818

3. European Association for the Study of the Liver EASL Clinical Practice Guidelines: Management of Hepatocellular Carcinoma. J Hepatol (2018) 69 (1):182-236. doi: 10.1016/j.jhep.2018.03.019

4. Llovet JM, Di Bisceglie AM, Bruix J, Kramer BS, Lencioni R, Zhu AX, et al. Design and Endpoints of Clinical Trials in Hepatocellular Carcinoma. J Natl Cancer Inst (2008) 100(10):698-711. doi: 10.1093/jnci/djn134

5. Shah SA, Cleary SP, Wei AC, Yang I, Taylor BR, Hemming AW, et al. Recurrence After Liver Resection for Hepatocellular Carcinoma: Risk Factors, Treatment, and Outcomes. Surgery (2007) 141(3):330-9. doi: 10.1016/j.surg.2006.06.028

6. Sempokuya T, Wong LL. Ten-Year Survival and Recurrence of Hepatocellular Cancer. Hepatoma Res (2019) 5:38. doi: 10.20517/2394-5079.2019.013

7. Schmidt-Wolf IG, Negrin RS, Kiem HP, Blume KG, Weissman IL. Use of a SCID Mouse/Human Lymphoma Model to Evaluate Cytokine-Induced Killer

\section{DATA AVAILABILITY STATEMENT}

The raw data supporting the conclusions of this article will be made available by the authors, without undue reservation.

\section{AUTHOR CONTRIBUTIONS}

$\mathrm{H}-\mathrm{LK}, \mathrm{E}-\mathrm{KL}$, and J-HL contributed to conception and design of the study. J-HL organized the database. H-LK, S-HK, and J-YC performed the statistical analyses and interpreted results. J-YC and $\mathrm{S}$-HK contributed equally to this work and deserve co-first authorship. $\mathrm{H}-\mathrm{LK}$ and J-HL are co-corresponding authors. All authors contributed to the article and approved the submitted version.

\section{FUNDING}

This study was supported by GC Cell Corp (Yongin, Korea). The sponsor was not involved in the study design, collection, analysis, interpretation of data, the writing of this article or the decision to submit it for publication.

\section{ACKNOWLEDGMENTS}

We would like to thank Editage (www.editage.co.kr) for English language editing.

\section{SUPPLEMENTARY MATERIAL}

The Supplementary Material for this article can be found online at: https://www.frontiersin.org/articles/10.3389/fonc.2021.728740/ full\#supplementary-material

Cells With Potent Antitumor Cell Activity. J Exp Med (1991) 174(1):139-49. doi: $10.1084 /$ jem.174.1.139

8. Zhang Y, Schmidt-Wolf IGH. Ten-Year Update of the International Registry on Cytokine-Induced Killer Cells in Cancer Immunotherapy. J Cell Physiol (2020) 235(12):9291-303. doi: 10.1002/jcp.29827

9. Lee JH, Lee JH, Lim YS, Yeon JE, Song TJ, Yu SJ, et al. Adjuvant Immunotherapy With Autologous Cytokine-Induced Killer Cells for Hepatocellular Carcinoma. Gastroenterology (2015) 148(7):1383-91.e6. doi: 10.1053/j.gastro.2015.02.055

10. Lee JH, Lee JH, Lim YS, Yeon JE, Song TJ, Yu SJ, et al. Sustained Efficacy of Adjuvant Immunotherapy With Cytokine-Induced Killer Cells for Hepatocellular Carcinoma: An Extended 5-Year Follow-Up. Cancer Immunol Immunother (2019) 68(1):23-32. doi: 10.1007/s00262-018-2247-4

11. Yoon JS, Song BG, Lee JH, Lee HY, Kim SW, Chang Y, et al. Adjuvant Cytokine-Induced Killer Cell Immunotherapy for Hepatocellular Carcinoma: A Propensity Score-Matched Analysis of Real-World Data. BMC Cancer (2019) 19(1):523. doi: 10.1186/s12885-019-5740-Z

12. Woods BS, Sideris E, Palmer S, Latimer N, Soares M. Partitioned Survival and State Transition Models for Healthcare Decision Making in Oncology: Where Are We Now? Value Health (2020) 23(12):1613-21. doi: 10.1016/j.jval.2020.08.2094

13. Marrero JA, Kulik LM, Sirlin CB, Zhu AX, Finn RS, Abecassis MM, et al. Diagnosis, Staging, and Management of Hepatocellular Carcinoma: 2018 
Practice Guidance by the American Association for the Study of Liver Diseases. Clin Liver Dis (Hoboken) (2019) 13(1):1. doi: 10.1002/cld.802

14. The Health Insurance Review and Assessment Service (HIRA) Guidelines for Economic Evaluation of Pharmaceuticals in Korea. Wonju: Health Insurance Review \& Assessment Service (2011).

15. Husereau D, Drummond M, Petrou S, Carswell C, Moher D, Greenberg D, et al. Consolidated Health Economic Evaluation Reporting Standards (CHEERS)-Explanation and Elaboration: A Report of the ISPOR Health Economic Evaluation Publication Guidelines Good Reporting Practices Task Force. Value Health (2013) 16(2):231-50. doi: 10.1016/j.jval.2013.02.002

16. Pollom EL, Lee K, Durkee BY, Grade M, Mokhtari DA, Wahl DR, et al. CostEffectiveness of Stereotactic Body Radiation Therapy Versus Radiofrequency Ablation for Hepatocellular Carcinoma: A Markov Modeling Study. Radiology (2017) 283(2):460-8. doi: 10.1148/radiol.2016161509

17. Ock M, Lim SY, Lee HJ, Kim SH, Jo MW. Estimation of Utility Weights for Major Liver Diseases According to Disease Severity in Korea. BMC Gastroenterol (2017) 17(1):103. doi: 10.1186/s12876-017-0660-3

18. Yang SY, Park SK, Kang HR, Kim HL, Lee EK, Kwon SH. Haematological Cancer Versus Solid Tumour End-of-Life Care: A Longitudinal Data Analysis. BMJ Support Palliat Care (2020). doi: 10.1136/bmjspcare-2020-002453

19. Hoyle MW, Henley W. Improved Curve Fits to Summary Survival Data: Application to Economic Evaluation of Health Technologies. BMC Med Res Methodol (2011) 11:139. doi: 10.1186/1471-2288-11-139

20. Peabody JW, Lee SW, Bickel SR. Health for All in the Republic of Korea: One Country's Experience With Implementing Universal Health Care. Health Policy (1995) 31(1):29-42. doi: 10.1016/0168-8510(94)00669-6

21. Kim L, Kim JA, Kim S. A Guide for the Utilization of Health Insurance Review and Assessment Service National Patient Samples. Epidemiol Health (2014) 36:e2014008. doi: 10.4178/epih/e2014008

22. Weinstein MC, Torrance G, McGuire A. QALYs: The Basics. Value Health (2009) 12(Suppl 1):S5-9. doi: 10.1111/j.1524-4733.2009.00515.x

23. Cadier B, Bulsei J, Nahon P, Seror O, Laurent A, Rosa I, et al. Early Detection and Curative Treatment of Hepatocellular Carcinoma: A Cost-Effectiveness Analysis in France and in the United States. Hepatol (Baltimore Md) (2017) 65 (4):1237-48. doi: 10.1002/hep.28961

24. Rognoni C, Ciani O, Sommariva S, Tarricone R. Real-World Data for the Evaluation of Transarterial Radioembolization Versus Sorafenib in Hepatocellular Carcinoma: A Cost-Effectiveness Analysis. Value Health (2017) 20(3):336-44. doi: 10.1016/j.jval.2016.09.2397

25. Tangka FK, Subramanian S, Sabatino SA, Howard DH, Haber S, Hoover S, et al. End-Of-Life Medical Costs of Medicaid Cancer Patients. Health Serv Res (2015) 50(3):690-709. doi: 10.1111/1475-6773.12259

26. May GS, DeMets DL, Friedman LM, Furberg C, Passamani E. The Randomized Clinical Trial: Bias in Analysis. Circulation (1981) 64(4):66973. doi: 10.1161/01.CIR.64.4.669

27. White DL, Thrift AP, Kanwal F, Davila J, El-Serag HB. Incidence of Hepatocellular Carcinoma in All 50 United States, From 2000 Through 2012. Gastroenterology (2017) 152(4):812-20.e5. doi: 10.1053/j.gastro.2016.11.020

28. Kennedy K, Graham SM, Arora N, Shuhart MC, Kim HN. Hepatocellular Carcinoma Among US and Non-US-Born Patients With Chronic Hepatitis B: Risk Factors and Age at Diagnosis. PloS One (2018) 13(9):e0204031. doi: 10.1371/journal.pone.0204031

29. National Cancer Institute Surveillance, Epidemiology, and End Results Program. Cancer Stat Facts: Liver and Intrahepatic Bile Duct Cancer.
(2020). Available at: https://seer.cancer.gov/statfacts/html/livibd.html [November 24. 2021].

30. Zhu RX, Seto WK, Lai CL, Yuen MF. Epidemiology of Hepatocellular Carcinoma in the Asia-Pacific Region. Gut Liver (2016) 10(3):332-9. doi: $10.5009 /$ gnl15257

31. Korean Liver Cancer Association; National Cancer Center. 2018 Korean Liver Cancer Association-National Cancer Center Korea Practice Guidelines for the Management of Hepatocellular Carcinoma. Gut Liver (2019) 13(3):227-99. doi: $10.5009 /$ gnl19024

32. Chon YE, Lee HA, Yoon JS, Park JY, Kim BH, Lee IJ, et al. Hepatocellular Carcinoma in Korea Between 2012 and 2014: An Analysis of Data From the Korean Nationwide Cancer Registry. J Liver Cancer (2020) 20(2):135-47. doi: 10.17998/jlc.20.2.135

33. Bruix J, Takayama T, Mazzaferro V, Chau GY, Yang J, Kudo M, et al. Adjuvant Sorafenib for Hepatocellular Carcinoma After Resection or Ablation (STORM): A Phase 3, Randomised, Double-Blind, Placebo-Controlled Trial. Lancet Oncol (2015) 16(13):1344-54. doi: 10.1016/S1470-2045(15)00198-9

34. Zhu XD, Li KS, Sun HC. Adjuvant Therapies After Curative Treatments for Hepatocellular Carcinoma: Current Status and Prospects. Genes Dis (2020) 7 (3):359-69. doi: 10.1016/j.gendis.2020.02.002

35. Seferina SC, Ramaekers BLT, de Boer M, Dercksen MW, van den Berkmortel F, van Kampen RJW, et al. Cost and Cost-Effectiveness of Adjuvant Trastuzumab in the Real World Setting: A Study of the Southeast Netherlands Breast Cancer Consortium. Oncotarget (2017) 8(45):79223-33. doi: 10.18632/oncotarget.16985

36. Millar JA, Millward MJ. Cost Effectiveness of Trastuzumab in the Adjuvant Treatment of Early Breast Cancer: A Lifetime Model. Pharmacoeconomics (2007) 25(5):429-42. doi: 10.2165/00019053-200725050-00006

37. Hisashige A, Sasako M, Nakajima T. Cost-Effectiveness of Adjuvant Chemotherapy for Curatively Resected Gastric Cancer With S-1. BMC Cancer (2013) 13:443. doi: 10.1186/1471-2407-13-443

Conflict of Interest: E-KL report receiving grants from Green Cross Cell Corp during the conduct of the study. J-HL reports a research grant and lecture fee from Green Cross Cell Corp. H-LK has received research grant and lecture fee from Green Cross Cell Corp.

The remaining authors declare that the research was conducted in the absence of any commercial or financial relationships that could be construed as a potential conflict of interest.

Publisher's Note: All claims expressed in this article are solely those of the authors and do not necessarily represent those of their affiliated organizations, or those of the publisher, the editors and the reviewers. Any product that may be evaluated in this article, or claim that may be made by its manufacturer, is not guaranteed or endorsed by the publisher.

Copyright (๑) 2021 Cho, Kwon, Lee, Lee and Kim. This is an open-access article distributed under the terms of the Creative Commons Attribution License (CC BY). The use, distribution or reproduction in other forums is permitted, provided the original author(s) and the copyright owner(s) are credited and that the original publication in this journal is cited, in accordance with accepted academic practice. No use, distribution or reproduction is permitted which does not comply with these terms. 